\title{
The Study of the Application of SNA in the Core Personality Words of Chinese New College Students
}

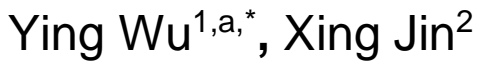 \\ ${ }^{1}$ School of Humanities and Social Sciences, Shanxi Medical University, Taiyuan, China. \\ ${ }^{2}$ Affiliated Tumor Hospital, Shanxi Medical University, Taiyuan, China. \\ awuguaiguai9896@163.com
}

Keywords: Personality words, Chinese new college students, social network analysis.

Abstract. Social network analysis (SNA) is one of the research methods of sociology, social construction is the main way to analyze the relationship between the social structure element, to analyze the global network and the individual network for the whole effect. Personality is an important research field of psychology. As a basic research tool, personality words are widely used in the research of personality psychology and social psychology. In this study, we extracted the personality words from the selected personality scales, carried on the questionnaire survey to the Chinese new college students, the personality words data obtained by the SNA software for social network analysis and then we found out the core words. From the results, we can see that new college students pay more attention to interpersonal communication and their moral cultivation. In addition, they are sometimes emotionally unstable. This study provides a basis for the prevention of mental illness and mental health education.

\section{武颖 $1, \mathrm{a},{ }^{*}$, 金星 ${ }^{2}$ \\ ${ }^{1}$ 山西医科大学人文学院, 太原, 中国 \\ ${ }^{2}$ 山西医科大学附属肿瘤医院, 太原, 中国 \\ awuguaiguai9896@163.com}

社会网络分析在大学新生人格核心词汇研究中的应用研究

关键词: 人格词汇; 中国大学新生; 社会网络分析

中文摘要. 社会网络分析法 (SNA) 作为社会学研究方法之一, 主要是采用社会建构的方式 分析社会结构元点之间的关系, 借此来分析这种全局网络以及个体网络对于整体的影响作用。 人格是心理学研究的重要领域, 人格词汇作为基础研究工具, 被广泛应用于人格心理学和社 会心理学的研究中。本研究从选定的人格量表中抽取人格词汇, 对大学新生进行问卷调查, 获得的人格词汇数据由SNA软件进行社会网络分析, 找出其中的人格核心词汇。从研究结果 可以看出, 大学新生比较注重人际交往和自身道德修养。另外, 他们有时情绪不稳定。本研 究对大学新生心理疾病的预防和心理健康教育提供了依据。

\section{1. 研究背景}

社会网络分析法（SNA）作为社会学研究方法之一，主要是采用社会建构的方式分析社 会结构元点之间的关系, 借此来分析这种全局网络以及个体网络对于整体的影响作用。SNA 强调行为主体之间 “关系” 的重要性, 构建网络的关系被看作是网络的结构, 因此SNA 是 一种结构分析 [1]。 
人格是心理学研究的重要领域, 人格特质词汇作为基础研究工具, 被广泛应用于人格心 理学和社会心理学的研究中。大多数的人格特质名称都会被编码到自然语言中去。因此, 自然 语言中包含了这一语言使用者的人格维度，同时，不同的语言也会对人格特点维度产生影 响。在西方从词汇入手研究人格维度已有较长的历史, 而从中文词汇入手的研究则是最近十 几年才开始的。

从词汇中研究人格维度的最早努力可以追溯到高尔顿爵士的由 1000 多个词组成的词单。 美国心理学家A11port和Odbest [2] 从1925年版的《韦氏国际字典》中挑出了17953个 “能区分 人类行为差异” 的术语为从词汇中研究人格维度提供了必要的基础。随后, Catte11[3]提出 了16个人格因素, Tupse和Christa1 [4] 提出了 “大五” 人格维度, 并得到很多心理学家的验 证。

最早研究中文人格木语的是台湾大学心理系的杨国枢 [5]和李本华，他们从小说、报纸以 及西方学者的词表中得到 900 个中文人格术语，压缩成557个术语，并对其好恶度、意义度、 以及熟悉度进行了评定。

十几年之后，北京大学心理系的研究者也采用相似的途径对 670 个中文人格特质形容词 进行了同样的评定。

社会网络分析法是一种社会学研究方法, 社会学理论认为社会不是由个人而是由网络构 成的, 网络中包含结点及结点之间的关系, 社会网络分析法通过对于网络中关系的分析探讨 网络的结构及属性特征。本文拟采用社会网络分析法对中国大学新生的人格核心词汇进行研 究。

\section{2. 材料方法}

本研究中采用的人格词汇来自五个成熟量表, 分别是: 五因素人格量表(NEO PI -R)、卡 特尔十六种人格因素检验 $(16 \mathrm{PF})$ 、加利福尼亚心理调查表 $(\mathrm{CPI})$ 、大五人格问卷 (CPFFI) 和 中国人人格量表 (QZPS), 共选出词汇118 个。由5名心理学院硕士研究生对词汇进行双向 翻译。

本研究选取医学院校261名新生进行问卷调查, 每名学生要求从118个词汇中选出最能代 表自己性格的 10 个词语。剔除无效问卷5份，回收有效问卷 256 份，有效率 $98.1 \%$ 。

获得的人格词汇数据经过加工, 处理, 选出频次最高的前 30 个词汇导入到Excel软件中制成共 现矩阵。再经由Ucinet、Netdraw等软件进行社会网络分析。

表 1 频次最高的前 30 个人格词汇

\begin{tabular}{|l|l|l|l|l|l|}
\hline 排名 & 人格词汇 & 频次 & 排名 & 人格词汇 & 频次 \\
\hline 1 & 重亲情 & 95 & 16 & 易于沟通 & 40 \\
\hline 2 & 善良 & 73 & 17 & 多愁善感 & 39 \\
\hline 3 & 友好 & 71 & 18 & 能容忍 & 39 \\
\hline 4 & 独立 & 62 & 19 & 感情丰富 & 38 \\
\hline 5 & 有责任感 & 56 & 20 & 理智 & 37 \\
\hline 6 & 重人情 & 54 & 21 & 性格内向 & 37 \\
\hline 7 & 爱幻想 & 53 & 22 & 相信事在人为 & 37 \\
\hline 8 & 乐观 & 53 & 23 & 坚持原则 & 37 \\
\hline 9 & 宽容 & 49 & 24 & 心直口快 & 35 \\
\hline 10 & 敏感 & 48 & 25 & 节俭 & 35 \\
\hline 11 & 真诚 & 46 & 26 & 知恩图报 & 34 \\
\hline 12 & 自觉性差 & 46 & 27 & 遵守纪律 & 31 \\
\hline 13 & 助人为乐 & 45 & 28 & 坚强 & 31 \\
\hline 14 & 人际关系和谐 & 44 & 29 & 工作认真 & 30 \\
\hline 15 & 重亲情 & 42 & 30 & 依赖 & 30 \\
\hline
\end{tabular}




\section{3. 结果分析}

\section{1 中心度分析}

图 1 为 30 个人格特质词汇的社会网络图谱。两点之间的连线值越大, 共现关系越强, 离得 也就越近，反之，两顶点之间的连线值越小，共现关系越弱，离得也就越远。我们用点的大 小代表该节点点度中心度的大小, 线条的粗细表示每一个词汇对之间的共现次数, 线条越粗, 表示两个词汇共现的次数越多。

中心度分析是社会网络分析方法最主要的分析工具, 中心度反映了行动者在网络中所处的地 位及权利影响。根据计算方法的不同, 中心度可以分为三种: 点度中心度, 中间中心度和接 近中心度。在一个社会网络中, 点度中心度等于一个节点所具有的连线数量。因此, 一个具 有高点度中心度的词汇必定与网络中的其他词汇有高度的直接联系, 这就意味着它是整个网 络的中心。

在网络中，中心度排名前十的词汇为重亲情、善良、友好、真诚、独立、有责任感、重 人情、宽容、爱幻想和敏感。说明这些词汇处于该网络的核心位置, 在权利、声望以及影响 方面较强。

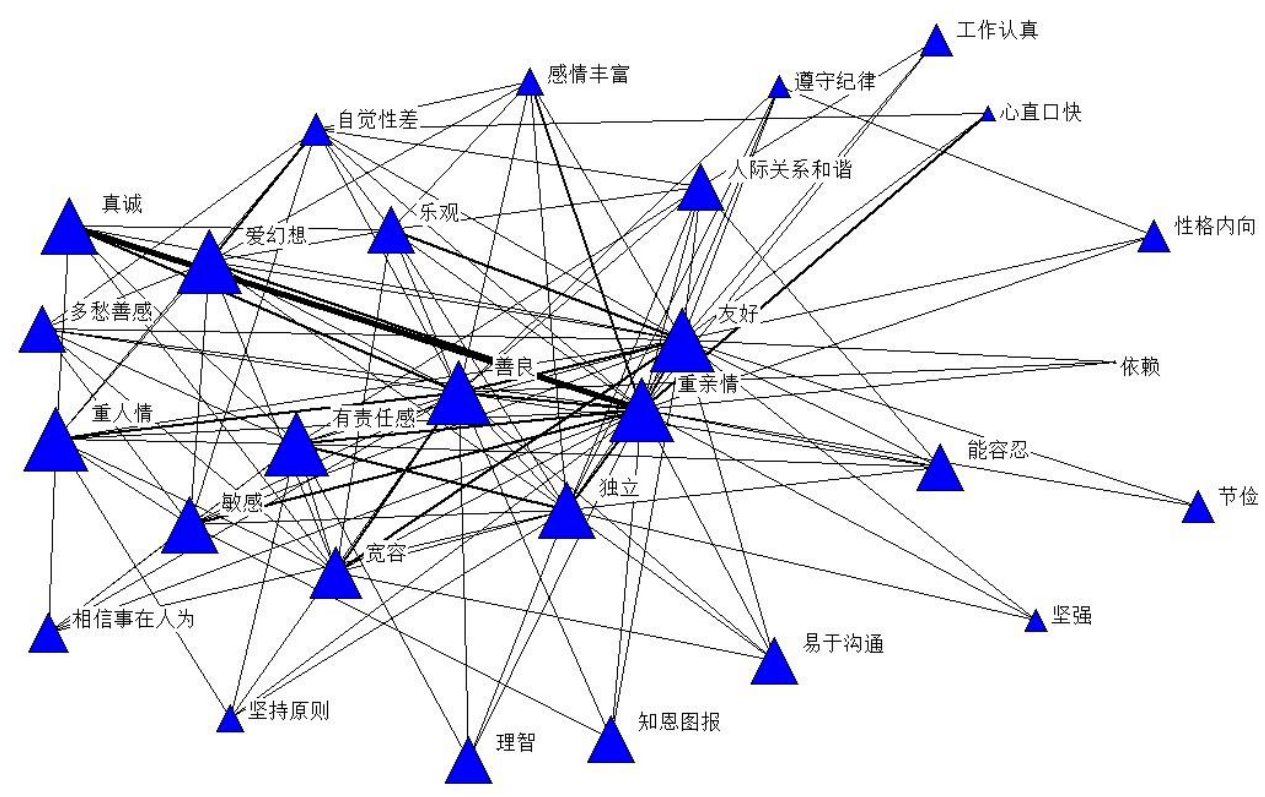

图1频次最高的前 30 个人格词汇的社会网络图谱

\section{2 核心边缘分析}

核心-边缘结构分析是对各种网络进行定量研究, 用于区分出网络中密度较高的一系列行 动者 (核心) 和密度较低的一系列行动者（边缘）。核心行动者在与边缘行动者的交换关系 中处于优势地位。

我们将这种方法应用于人格特质词汇社会网络的分析, 利用UCINET6.0, 计算出核心-边 缘的相关值为 0.712 。从图2中我们可以看出两极分化的核心-边缘结构非常明显, 分核心词汇, 边缘词汇两部分。核心词汇为: 重亲情、善良、友好、独立、有责任感、重人情、爱幻想、 乐观、宽容、敏感和自觉性差。 


\section{$3.3 \mathrm{~K}-$ 丛分析}

$\mathrm{k}$-从是指满足下列条件的一个凝聚子群，即在这样一个子群中，每个点都至少与除了 $\mathrm{k}$ 个 点之外的其他点直接相连。下面是对本文构建的词汇网络进行 $\mathrm{k}$-从丛分析的结果, $\mathrm{k}$ 的取值为 2 , 共找到 588 个 2 -丛。其中，友好、有责任感、重人情、善良、重亲情、爱幻想6个词为所有2从的共有组成因素。

\section{4. 讨论}

本研究采用社会网络分析法对大学新生人格特质词汇进行了三方面的研究。其中，重亲 情、善良、友好、有责任感、重人情、爱幻想 6 个词在三部分研究中都处于核心位置, 独立、 宽容、敏感 3 个词在中心度分析和核心-边缘分析中处于核心位置, 真诚 1 个词只在中心度分析 中处于核心位置，自觉性差1个词只在核心-边缘分析中处于核心位置。

从以上人格特质核心词汇可以看出, 首先, 医学大学生注重人际交往, 体现出以关系为自我 依托的文化特征, 这可能与中国人重视和谐, 处事方式以关系为导向有关。其次, 道德感作 为中国人自我完善的品质一直为社会所重视, 医学大学生同样注重自身道德修养。另外, 责 任感作为一项重要的工作人格特征也受到医学大学生的重视, 但是负性词汇的存在提示责任 感水平有待进一步提高。最后, 医学大学生尚未进入社会, 对自身发展难免理想化, 有时情 绪不稳定, 这也符合青年人的人格特征。本研究结果中既包含了人类共性的人格成分, 也体 现出中国文化和医学大学生特有的人格因素。

人格是个体行为的全部本质, 不良的人格特征是产生疾病的危险因素。因此, 对人格的研究 不仅可以预防疾病, 还能为学生心理健康教育提供依据。

\section{致谢}

本文为国家自然青年基金项目《精神医学领域科研合作及研究前沿趋势预测》(71503152) 的阶段性成果之一。

\section{References}

[1] Wasserman, Stanley \& Katherine Faust. Social Network Analysis: Methods and Applications, Cambridge University Press, pp. 5-20, 1994.

[2] Allport GW, Odbert HS. Trait names: A psycho-lexieal study. Paychological Monographs, vol. 41, 1936.

[3] Cattel, RB., Eber, HE., \& Tatsuoka, MM. Handbook for the Sixteen Personality Factor Questionnaire (16PF). Champaign, IL: IPAT, 1970.

[4] Tupes EC, Christal RC. Recurrent personality factor based on trait ratings. Lackland Air Force Base, T X: U. S. Air Force, 1961.

[5] Yang KS, Bond MH. Exploring implicit personal theories with indigenious or imported constructs: The Chinese case. Journal of Personality and Social Psychology, vol. 6, pp. 1087-1095, 1990. 